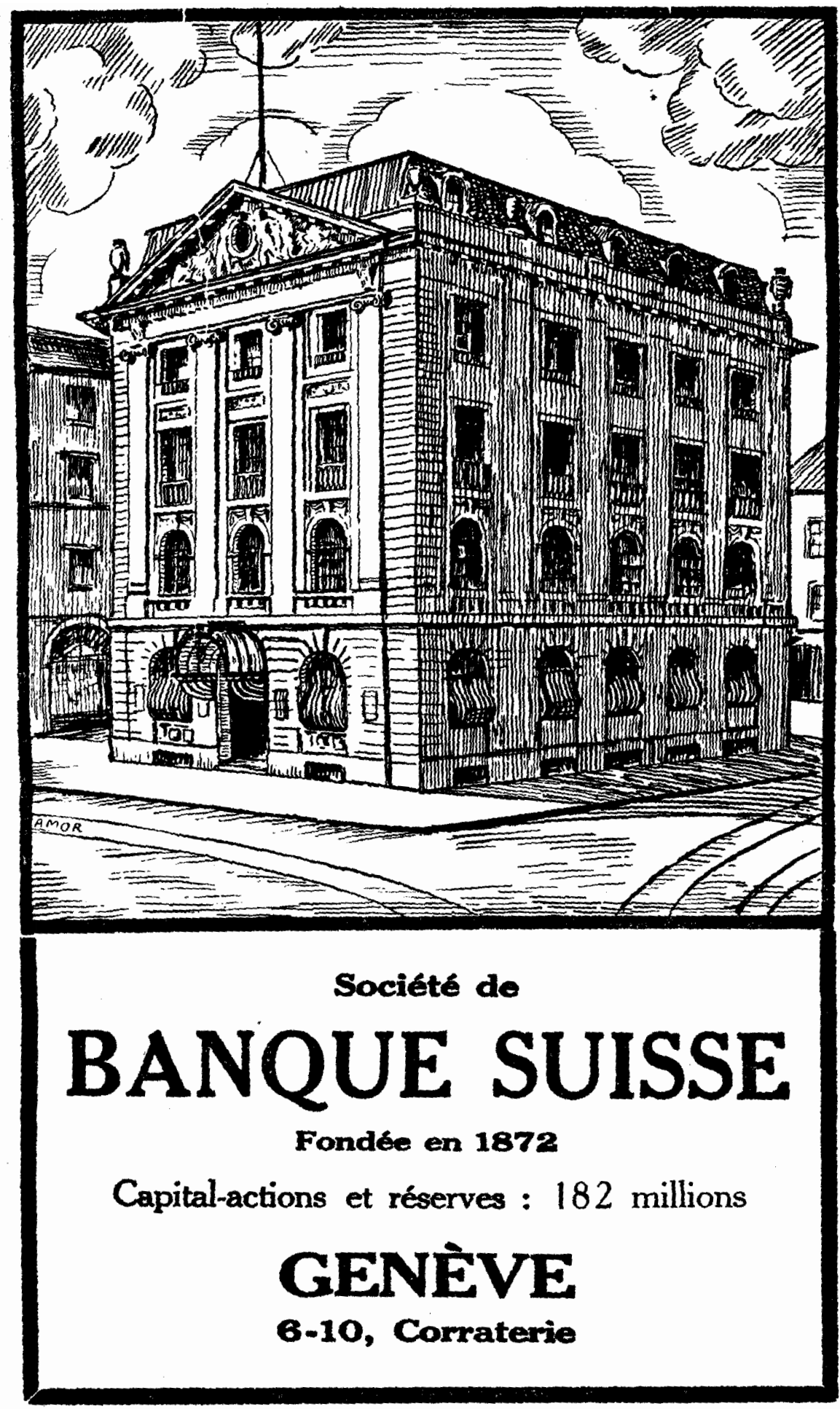




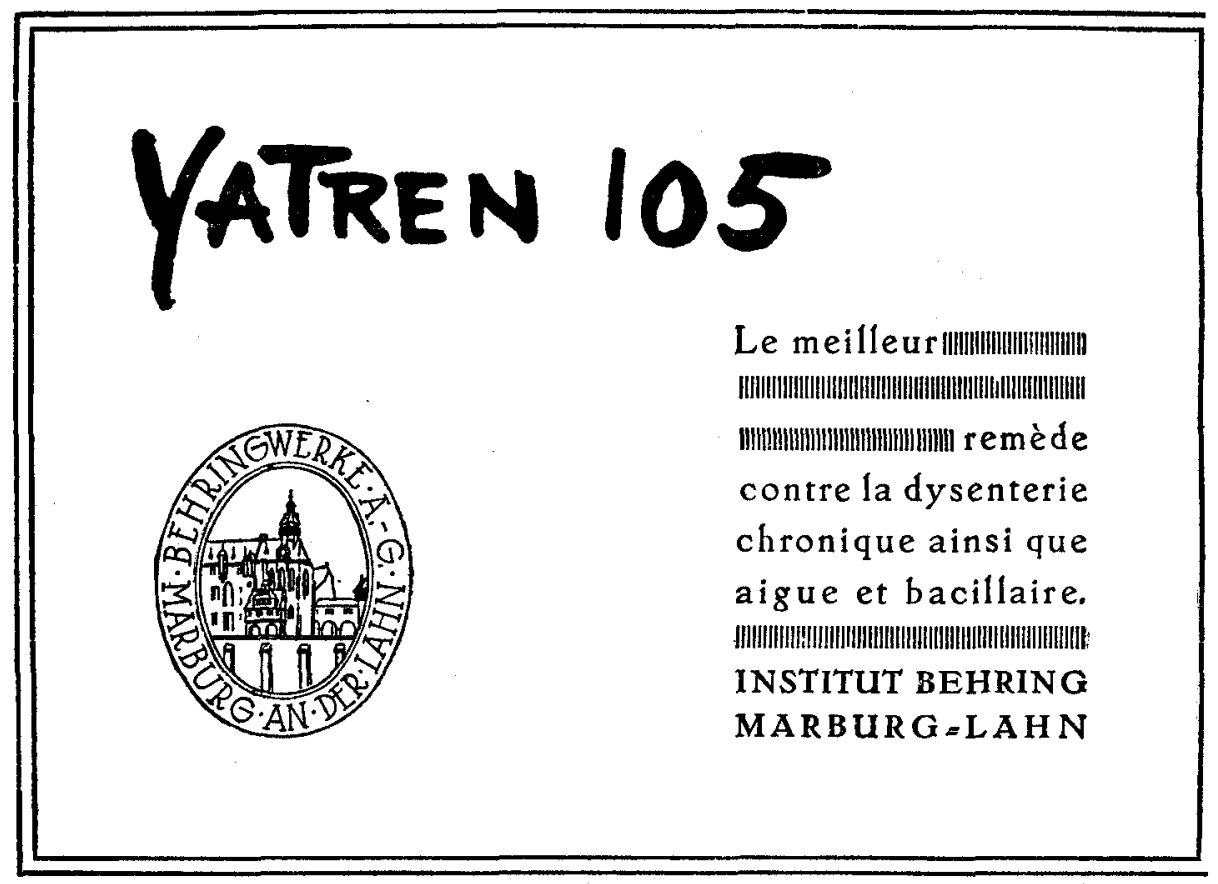

\section{REVUE \\ INTERNATIONALE DE L'ENFANT}

31, quai du Mont-Blanc, GENĖVE

Le numéro : 1 fr. suisse. 12 numéros (un an) : 10 fr. suisses

Sommaire du numéro de février 1929 :

A la mémoire d'Eglantine Jebb.

Schwererziehbare in der Fürsorgeerziehung, von Justus Erhardt, Berlin.

Children of the Future, by Gertrude Eaton, vice-chairman of the Howard League for Penal Reform.

La récupération fonctionnelle des paralytiques par l'appareillage, par le Dr G. Bidou, chef du Laboratoire de récupération fonctionnelle des hôpitaux de la Salpêtrière, médecin en chef de l'hôpital Sainte-Isabelle.

Ueber die Bedeutung der Schilddrüse für das Kind, von Prof. $D^{r}$ Edmund Nobel, Universitäts-Kinderklinik, Vienne.

Chronique. - Nouvelles de l'U.I.S.E. -- Bibliographie. 


\section{Banque Ottomane}

Capital Lstg. 10.000.000.- dont molite versée.

Sì̀ge central à Constantinople, GALATA.

Agences a Péra et Stamboul, ainsi que dans les principales localités de la Turquie, l'Egypte, Chypre, la Palestine, la Mésopotamie, la Perse et la Grèce.

Londres : 26, Throgmorton Street, E.C.2.,

Manchester : 56/60, Cross Street, à Paris : 7, rue Meyerbeer (90 Arr.), à Marseille : 41 et 43 , rue Grignan,

à Tunis : 68, Avenue Jules Ferry.

Se charge de toutes opérations de Banque.

Filiale pour la Syrie : Banque de Syrie et du Grand Liban.
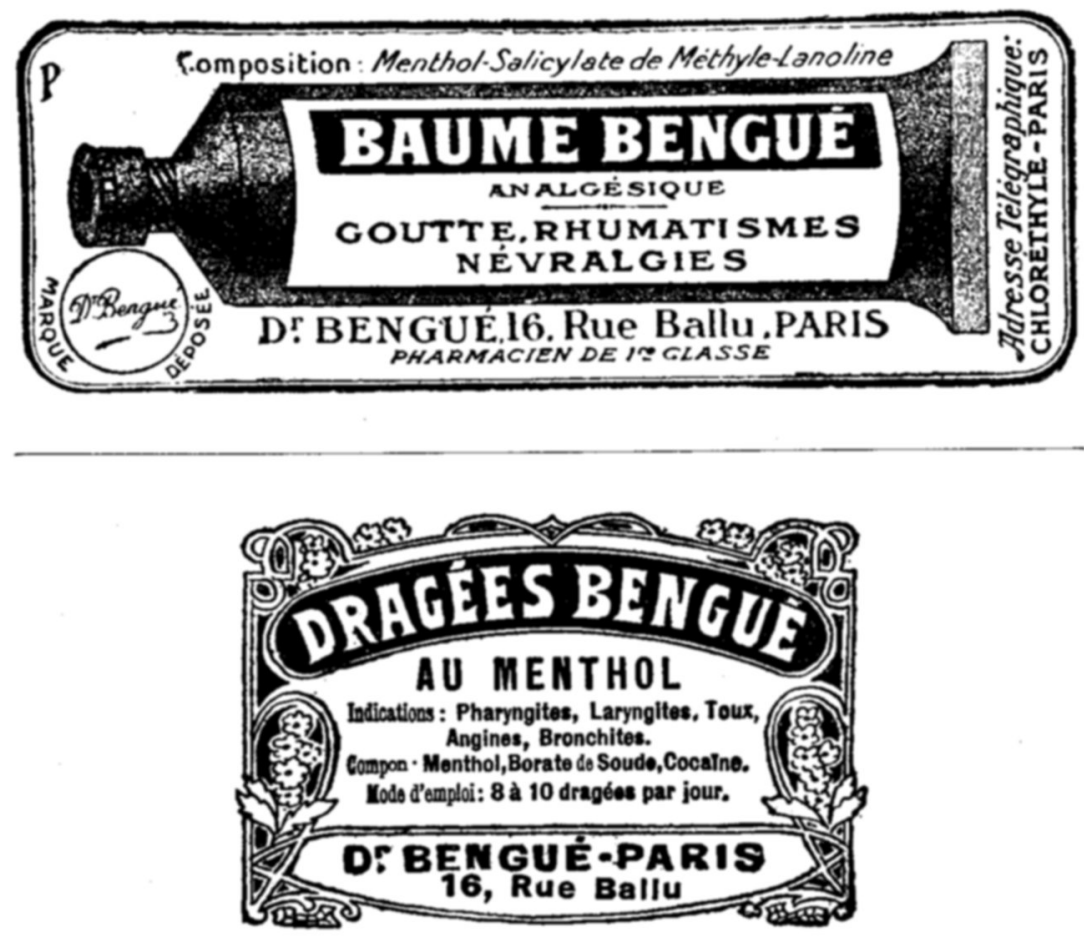
Editeurs : FELIX ALCAN, Paris - NICOLA ZANICHELLI, Bologne - WILLIAMS \& NORGATE, Londres. - AKAD. VERLAGSGESELLSCHAFT, Leipzig - G. E. STECHERT \& Co., New York. - RUIZ hERMANOS, Madrid. - RENASCENÇA PORTUGUESA, Porto - THE MARUZEN COMPANY, Tokio.

\section{"SCIENTIA"}

REVIE WTERNATIONALE OE SYHTHESE SEIENTIPATR Paralssant mensuellement (en faseicule de 100 à l20 pages ghactin) Directeur : Eugenio RIGNANO

Est l'unique Revue à collaboration vraiment internationale.

Est l'unique Revue à diffusion absolument mondiale.

Est l'unique Revue de synthèse et d'unification du savoir, qui traite les questions fondamentales de toutes les sciences : histoire des sciences, mathématiques, astronomie, géologie, physique, chimie, biologie, psychologie et sociologie.

Est l'unique Revue par conséquent qui, intéressant directement les statisticiens, les démographes, les ethnographes, les économistes et ceux qui cultivent le droit, l'histoire des religions et, d'une façon générale, la sociologie, par ses nombreux et importants articles et comptes rendus relatifs à ces sciences, leur offre anssi le moyen de connaitre sous une forme résumée et synthétique, les plas grands problèmes de toutes les antres branches du savoir.

Est l'unique Revue qui puisse se vanter d'avoir parmi ses collaborateurs les savants les plus illustres du monde entier. Une liste de ceux-ci, comprenant plus de 350 noms, est reproduite dans presque tous les fascicules.

Les articles sont publiés dans la langue de leurs auteurs, et à chaque fascicule est joint un supplément contenant la traduction francaise de tous les articles non français. Ainsi la revue est complètement accessible même à qui ne connaît que la langue française. Demandez un numéro spécimen gratuit au Secrétaire Général de "Scientia", Milan, en joignant à la demande, pour remboursement des frais d'envoi, la somme de quatre francs en timbres-poste de votre pays.

ABONNEMENT: Lire 200.

BUREAUX DE LA ReVue : 12, vis A. de Togni - Milano (16).

Secrétaire Général de la Rédaction : D* Paolo BONETTI.

\section{BULLETIN}

DE L'UNION DES FEMMES DE FRANCE (CROIX-ROUGE FRANÇAISE)

\section{Bulletin mensuel}

Abonnement d'un an : 5 francs

Pour faire partie de l'Union des Femmes de France, il suffit de faire parvenir au Siège Central de l'Union des Femmes de France, 102, Boulevard Malesherbes, à Paris, une des cotisations suivantes :

de 5 à 10 francs comme Membre adhérent;

de 10 à 30 francs comme Membre titulaire.

Rachat de cotisation :

Membre bienfaiteur : 1000 francs. - Membre perpétuel : 200 francs. 
DE

\title{
DROIT INTERNATIONAL
}

De sciences diplomatiques et politiques

\section{(THE INTERNATIONAL LAW REVIEW)}

\author{
Organe officiel de "l'International Law Association"
}

fondée à GENÈVE en 1923 par Antoine SOTTILE, sous le patronage et avec la collaboration do:

L. L. E. N. Politis, E. Benès, H. Bellot, Phillimore, Adatci, Lord Cecil, De la Barra, B. Mussolini, G. Motta, C. R. Pusta, van Eysinga, C. de la Torriente, E. Rolin, Jaequemyns, P. Fauchille, A. Alvarez, J. Brown Scott, Ch. Lyon-Caen, Rouard de Card, A. Weiss, A. de La Pradelle, Merignhac, D. Anzilotti, de Montluc, B. C. J. Loder, M. Haber, D. J. Jitta, E. Borel, J. Valery, A. Prudhomme, Olivart, K. Neumeyer, Triepel, Schücking, Erich, Strupp, Institut Ibero-Américain de droit comparé, International Law Association, Institut de Droit International (Rome), etc., est la SEULE Revue paraissant en Suisse en matière de Droit International, de Sciences diplomatiques et politiques. Elle préconise la rénovation du droit international, la renaissance de la justice mondiale, la souveraineté effective du droit, la solidarité internationale, la morale dans la politique internationale, le développement de l'esprit international, le règlement pacifique des conflits, la défense des droits des petits Etats.

Paraissant au siège de la Société des Nations, la a REVUE DE D ROIT INTE RNATIONAL ø est à même de faire rapidement connaître et d'apprécier avec sûreté les règles que stipulera la communauté des Nations.

La REVUE DE DROIT INTERNATIONAL paraît tous les trois mois en livraisons de 70 a 120 pages. Les articles sont publiés dans la langue de leurs auteurs.

Numéro spécimen (arriéré) contre envoi de fr. 2.90 suisses.

ABONNEMENT : Suisse Fr. 30.- ; Union postale (francs suisses) Fr. 35. Tous les abonnements sont annuels et partent du numéro de Janvier. Prix spécial pour les pays à change déprécié. Les abonnements se palent à l'avance. (La Revue est honorée de souscriptions des Gouvernements.)

Pour les Membres de l'a International Law Association ", prix d'abonnement : fr. suisses 25.

Directeur :

\section{Dr Juris A. SOTTILE}

Chargé d'Affaires, Délégué permanent de Nicaragua à la Société des Nations Consul de la République de Libéria et de la Rép. de Nicaragua Membre du Conseil de I'Institut de Droit international de Rome.

Direction, Rédaction et Administration :

Rue Merle-d'Aubigné, 3 - GENÈVE (Suisse) 


\title{
LE BULLETIN ILLUSTRÉ
}

DE

\section{L'ASSOCIATION DES DAMES FRANÇAISES}

\author{
Croix-Rouge française
}

Paraît tous les deux mois : il rend compte de l'activité générale de l'Association; de nombreuses photographies accompagnent le texte $\left(34^{\mathrm{e}}\right.$ année) $)$

Abonnement d'un an, envoi franco : 5 francs.

Pour faire partie de l'Association des Dames Françaises, il suffit de faire parvenir au Siège central de l'A. D. F., 12, rue Gaillon, à PARIS, une des cotisations annuelles suivantes :

de 1 à 5 francs comme membre adhérent.

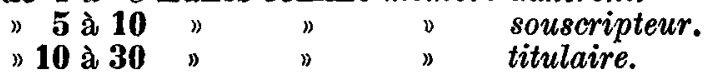

Les Membres titulaires peuvent racheter leur cotisation moyennant un versement unique de 300 francs.

\section{"WINTERTHOUR"}

Société Suisse d'Assurance

contre les Accidents

Assuranges :

Accidents de tous genres.

Responsabilité Civile vis-à-vis des Tiers. Vol.

Automobiles.
Société d'Assurance sur la Vie

\section{Assurances Vie}

toutes combinaisons, avec et sans participation aux bénéfices.

Rentes viagères.

\section{Direction des deux Sociétés à Winterthour}

Renseignements et prospectus gratuits par les

Agence générale

pour la branche Accidents :

Robert MEYER

Bureau national d'assurances

successeur ROESSIIIGER \& BOUET, GEnève
de

11, rue Géneral Dufour
Agence générale pour la branche Vie :

Louis GRILLET GENÈE

2. arenue Pictet-de-Rochemont 


\section{Adresses des Comités centraux (suite).}

* BELGIQUE. - Croix-Rouge de Belgique, rue de Livourne, 80, Bruxelles. BOLIVIE. - Croix-Rouge bolivienne, Casilla de Correo, 504, La Paz.

BRESIL. - Croix-Rouge brésilienne, Praça Vieira Souto, 10, Rio de Janeiro.

BULGARIE. - Croix-Rouge bulgare, 19, Bd. Totleben, Sofia.

CANADA. - Croix-Rouge canadienne, 410, Sherbourne Street, Toronto.

CHILI. - Croix-Rouge chilienne, Correo, 8, Casilla 7567, Santiago de Chile.

CHINE. - Croix-Rouge chinoise, Pélkin et 26, Kiukiang Road, Shanghaï.

COLOMBIE. - Croix-Rouge colombienne, Bogota.

COSTA-RICA. - Croix-Rouge costaricienne, Calle 5. Sur., San José.

CUBA. - Croix-Rouge cubaine, Ignacio Agramonte et av. de Belgica, La Havane.

DANEMARK. - Croix-Rouge danoise, Ved Stranden, 2, Copenhague, K.

DANTZIG. - Croix-Rouge de Dantzig, Neugarten, 12-16, Dantzig.

REPUBLIQUE DOMINICAINE. - Croix-Rouge dominicaine, St-Domingue.

EGYPTE. - Croissant-Rouge égyptien, Ismail Pacha, 8 (Garden City) Le Caire.

EQUATEUR. - Croix-Rouge équatorienne, Venezuela 46, Quito.

ESPAGNE. - Croix-Rouge espagnole, Calle de Sagâsta, 10, (Estafeta, 4). Madrid.

ESTONIE. - Croix-Rouge estonienne, Niguliste Tän, 12, Reval.

ETATS-UNIS. - Croix-Rouge américaine (Central Committee of the American Nationa

Red Cross), National Headquarters, Washington, D.C.

FINLANDE. - Croix-Rouge de Finlande, 16, Annankatu, Helsingiors.

FRANCE. - Croix-Rouge française, rue François ler, 21, Paris, $8 \mathrm{me.}$

** GRANDE-BRETAGNE. - Croix-Rouge britannique (British Red Cross Society), Berkeley Street 19, London W. 1.

GRECE. - Croix-Rouge hellénique, rue Solon, 39, Athènes.

GUATEMALA. - Croix-Rouge guatémalienne, 13, Calle Oriente, 2, Guatémala.

HONGRIE. - Croix-Rouge hongroise, Baross utca 15, Budapest, VIII.

INDES. - Croix-Rouge des Indes (Indian Red Cross Society), New-Dehli.

ISLANDE. - Croix-Rouge islandaise, Reyljavil.

ITALIE. - Croix-Rouge italienne, via Toscana, 12, Rome (25).

JAPON. - Croix-Rouge japonaise, 5 Shiba Park, Tokio.

LETTONIE. - Croix-Rouge lettone, Skolas ielá, 1, Riga.

LITHUANIE. - Croix-Rouge lithuanienne, Kestucio g-vé 8, Kaunas.

LUXEMBOURG. - Croix-Rouge luxembourgeoise, Luxembourg.

MEXIQUE. - Croix-Rouge mexicaine, 2, a de San Geronimo, 14, Mexico, D.F.

NORVEGE. - Croix-Rouge norvégienne, Grev Wedels Plass 5, Oslo.

PANAMA. - Croix-Rouge de Panama, Panama.

PARAGUAY. - Croix-Rouge paraguayenne, Avenida Espana, 505, Assomption.

*** PAYS-BAS. - Croix-Rouge néerlandaise, Princessegracht, 27, La Haye

PEROU. - Croix-Rouge péruvienne, Melchormalo 349, Lima.

PERSE. - Lion et Soleil-Rouges de Perse, Téhéran.

POLOGNE. - Croix-Rouge polonaise, rue Smolna, 6, Varsovie.

PORTUGAL. - Croix-Rouge portugaise, 1, Praça do Commercio, Lisbonne.

ROUMANIF. - Croix-Rouge roumaine, 40, rue N. Balcesco, Bucarest.

SALVADOR. - Croix-Rouge de Salvador, 13 a Avenida Norte y 9 a calle Poniente, San Salvador.

ROYAUME S.C.S. - Croix-Rouge des Serbes, Croates et Slovènes, Simina ulica, 21, Belgrade. SIAM. - Croix-Rouge siamoise, Bangkok.

SUEDEE. - Croix-Rouge suédoise (Svenska Roda Korset), Artillerigatan, 6, Stockholm, 14. SUTSSE. - Croix-Rouge suisse, Taubenstrasse, 8, Berne.

TCHÉCOSLOVAQUIE, - Croix-Rouge tchécoslovaque, Neklanova ul, 147, Prague VI. TURQUIE. - Croissant-Rouge turc, Angora.

**** U.R.S.S. (Union des républiques soviétiques socialistes). - Alliance des sociétés de la

Croix-Rouge et du Croissant-Rouge de l'U.R.S.S., Tverskaja-Yamskaja $n^{0} \mathbf{l} / \mathbf{3}, \mathbf{M}$ oscou. URUGUAY. - Croix-Rouge uruguayenne, Piedad 13.779, Montevideo.

VÉNÉZUELA. - Croix-Rouge vénézuélienne, Muñoz a Piñango, 10, Garacas.

* Section: Croix-Rouge du Congo, rue de Livourne, 80, Bruxelles.

* Awienne Croix-Rouge néo-zélandaise, P.O. Box 969, Wellington, N.Z.

** Branche: Croix-Rouge des Indes neerlandaises, Wellureden (Java).

*** Membres Croix-Rouge arménienne, Erivan.

de Croissant-houge de l'Azerbéidjan, rue Zévine, 1, Bakou.

l'Alliance : Croix-Fouge gếrorienne, 15 , rue Krylov, Tiflis.

Croix-Rouge russe (R.S.F.S.R.), Pouchetchnaia, 5, Mosrou

Croix-Rouge de la Russie Blanche, 3, rue Marx, Minsh.

Croissant-Rouge du Turkménistan, Polloratzk

Croix-Rouge ukranienne, 7 , rue du Théatre, Kharkov.

Croissant-Rouge de l'Uzbélistan, ul. Respublikanskaia, 13, Samarkand. 


\title{
Sommaire du Bulletin
}

\author{
T. LX, $\mathbf{N}^{\circ} 318$
}

FÉvrier 1929

Comité international de la Croix-Rouge. - Reconnaissance de la Croix-Rouge des Indes (Indian Red Cross Society) $\left(285^{\mathrm{e}}\right.$ circulaire), 127. - Publication conjointe (Annuaire 1929), 128.

Institut international d'études de matériel sanitaire. - Contributions à l'Institut de matériel sanitaire, 129.

Ligue. - Publication conjointe, 130. - Publication, 130. - Conférence de la Croix-Rouge de la jeunesse, 132.

Autriche. - Assemblée générale de la Croix-Rouge autrichienne, 133.

Brésil. - Mort du président de la Croix-Rouge brésilienne, 134.

Espagne. - Inauguration d'un dispensaire de la Croix-Rouge à Madrid, 135.

France. - La Croix-Rouge française chez les "mal lotis ", 136.

Hongrie. - La Croix-Rouge hongroise et l'Union internationale de secours, 137.

Italie. - Loi instituant la journée de la Croix-Rouge italienne et autorisant l'augmentation du prix des billets au profit de la Société, 138.

Royaume des Serbes, Croates et Slovènes. - Ecole d'infirmières de la Croix-Rouge des Serbes, Croates et Slovènes à Belgrade (hors-texte), 139.

Suisse. - Mort de Mile Alice Favre, membre d'honneur de la Direction de la Croix-Rouge suisse, 141.

Uzbékistan. - Trois ans d'activité de la Société du Croissant-Rouge de l'Uzbékistan (hors-texte), 142.

La Revue internationale de la Croix-Rouge, Bulletin international des Sociétés de la Croix-Rouge, paraît à la fin de chaque mois.

Prix, franco, un an: Fr. 20.- Le numéro: Fr. 2.Années 1919, 1920 et 1921 (rares): Fr. 30.-Administration : Promenade du Pin, 1, Genève. 